\title{
Assessing arrhythmia risk in a 15-year-old boy whose father died suddenly
}

\author{
Paul Dorian MD, Andrew D. Krahn MD
}

Competing interests: None declared.

This article has been peer reviewed.

The clinical scenario is fictional.

Correspondence to: Paul Dorian,dorianp@smh.ca

CMAJ 2016. DOI:10.1503 /cmaj.151365
A 15-year-old boy is referred for assessment of arrhythmia risk. The boy's father died suddenly six weeks ago. He was 43 years old, had been in excellent health, completely asymptomatic, and had exercised regularly. He went out for a run and was found on the trail without vital signs and with no apparent injury. The son is an elite soccer player, playing for a regional competitive team, with aspirations to play at the highest level.

\section{What further information is needed?}

Information from an autopsy on the father, if conducted, is crucial, because a small proportion of disorders leading to sudden death can be associated with heritable structural heart disease, such as hypertrophic cardiomyopathy, right ventricular cardiomyopathy and dilated cardiomyopathy (Boxes 1 and 2, and Appendix 1 at www.cmaj.ca/ lookup/suppl/doi:10.1503/cmaj.151365/-/DC1). ${ }^{1-4}$

Other structural disorders may not be heritable but can be identified at autopsy. Previously unrecognized coronary artery disease is the most common cause of exercise-related sudden death in people over 35 years old. ${ }^{4}$ Other conditions include anomalous origin of the coronary arteries, myocarditis, sarcoidosis and other rare infiltrative disorders (Box 1).

Primary arrhythmia syndromes without structural heart disease are associated with a normal cardiac autopsy and are often heritable. These include long and short QT syndromes, catecholaminergic polymorphic ventricular tachycardia and Brugada syndrome (Box 1). ${ }^{2}$

Obtaining a thorough family history is essential: a family history of unexpected sudden death, unusual syncope (e.g., with exercise), drowning, crib death or suspicious death related to a singlevehicle crash may be a clue to heritable arrhythmia. Frequently, sudden unexpected death in a relative will have been labelled a "heart attack" without detailed documentation. All unexpected out-of-hospital sudden deaths in any first-degree relative should be presumed arrhythmic until proven otherwise (Box 2). ${ }^{1}$

In the son, a history of loss of consciousness or near loss of consciousness, particularly during or immediately after exercise, may suggest serious arrhythmias. A history of apparent seizures may also represent arrhythmia, because secondary seizures frequently accompany cerebral hypoperfusion during arrhythmia. ${ }^{4}$ Sudden unexplained dyspnea or chest pain may be due to structural heart disease, such as hypertrophic cardiomyopathy or anomalous origin of a coronary artery (Box 2).

A thorough physical examination, including assessment of blood pressure and postural changes in pressure, auscultation for murmurs and peripheral pulses can reveal structural heart disease, such as coarctation of the aorta, valvular disease and cardiomyopathy (Box 2).3,4

\section{Are any investigations required?}

Based on observational studies and expert opinion (as detailed in guidelines ${ }^{2,4}$ ), investigations should be specifically directed at possible diagnoses (Boxes 1 and 2). A 12-lead electrocardiogram (ECG) should be carefully examined for evidence

Box 1: Possible cardiac causes of sudden death $^{1-3}$

- Coronary artery disease

- Cardiomyopathy

- Arrhythmogenic right ventricular dysplasia

- Hypertrophic cardiomyopathy

- Dilated cardiomyopathy

- Ion channelopathy

- Long and short QT syndrome

- Catecholaminergic polymorphic ventricular tachycardia

- Brugada syndrome

- Other

- Myocarditis

- Infiltrative cardiomyopathy (e.g., sarcoidosis) 
of repolarization abnormalities (Q-T interval), ST-segment and T-wave abnormalities (suggesting left ventricular hypertrophy, Brugada syndrome or hypertrophic cardiomyopathy), early repolarization or epsilon waves suggesting right ventricular cardiomyopathy. ${ }^{1,3}$ The ECG findings are often abnormal in high-performance athletes; sinus bradycardia, first-degree atrioventricular block, incomplete right bundle branch block, and left ventricular hypertrophy identified by voltage criteria alone are common and not usually a cause for concern. Echocardiography is recommended, specifically to assess the presence of hypertrophic or other forms of unrecognized cardiomyopathy (Box 2). ${ }^{4}$

An exercise stress test is particularly of interest for this patient, because his father died during exercise. It should be performed to look for exerciseinduced ventricular tachycardia. If polymorphic or bidirectional ventricular tachycardia occurs with exercise, this suggests catecholaminergic polymorphic ventricular tachycardia. If monomorphic, and of right ventricular origin, this suggests right ventricular cardiomyopathy. A normal Q-T interval at rest does not rule out long QT syndrome. The Q-T interval may paradoxically increase on standing or fail to shorten appropriately with exercise, with high specificity for long QT syndrome. ${ }^{5}$

In an asymptomatic person, Holter monitoring may be performed to identify ambient ventricular ectopy, often seen in people with cardiomyopathy. ${ }^{1}$

\section{Should the patient be referred?}

It is generally useful for a patient in this circumstance to be referred to a consultant with special interest and expertise in arrhythmia evaluation and treatment. ${ }^{4}$ First-degree relatives of young people $(<50 \mathrm{yr})$ who die suddenly and unexpectedly of apparent arrhythmia or of unknown cause should be referred for evaluation by a consultant. Regional multidisciplinary clinics, led by heart rhythm specialists, are being established that are well suited to evaluate patients in this circumstance (a list of clinics in Canada is available from the Canadian Genetic Heart Rhythm Network, www.heartrhythmresearch.ca). Specialized tests, including a signal-averaged ECG, cardiac magnetic resonance imaging, and epinephrine and procainamide infusions to uncover latent arrhythmia syndromes, may be performed in the clinics. ${ }^{1-3}$

These specialized clinics are also experienced at dealing with family support and education, as well as providing detailed explanations of potential risks for the short- and long-term future, and advice about competitive sport. Any impact on this patient's athletic aspirations will stem from results of cardiac investigations. ${ }^{4}$

\section{What should the patient be told about physical activity while investigation results are pending?}

If the patient has not had any of the symptoms noted above and his ECG findings are normal, he should be reassured and permitted any sport activity. However, he should be informed that, although unlikely, this advice may change after the results of investigations become available. For example, if the patient is found to have a condition associated with exertion-related events (e.g., hypertrophic cardiomyopathy), he may be advised not to participate in competitive sport. ${ }^{4}$

\section{Case revisited}

The patient was referred to a specialized arrhythmia clinic. There was no other relevant family history. The patient had no symptoms; findings on physical examination were unremarkable, and results of the initial investigations (ECG, echocardiogram and exercise stress test) were normal. Based on this information, the patient was reassured and continued to play soccer competitively. Three months later, the autopsy results on the father showed severe proximal narrowing of the left anterior descending coronary artery, with a fresh thrombus. The patient was counselled about the potential long-term risk of coronary artery disease and the importance of long-term monitoring for elevated cholesterol, diabetes and hypertension.

\section{Box 2: Assessment in cases of sudden} death ${ }^{1,3 *}$

\section{Sudden death victim}

- Family history: sudden death, drowning, single-vehicle crash, crib death, heart failure, "heart attack"

- Personal history: syncope, seizures, exercise intolerance, chest pain, medications

- Event history: exercise or rest, recent illnesses, fever, new medication

- Autopsy: preliminary and final report, molecular autopsy if no anatomic cause

\section{First-degree relative}

- Personal history: syncope, seizures, palpitations, chest pain, shortness of breath

- Physical examination: murmur, abnormal pulses

- Electrocardiography: electrocardiogram (ECG), high precordial lead ECG, signal-averaged ECG, exercise test, Holter monitor

- Imaging: echocardiography

- Special testing: drug challenge, cardiac magnetic resonance imaging, genetic testing if victim had a positive result from genetic testing for a relevant heritable disorder

*Typical ECG abnormalities found in selected conditions are shown in Appendix 1 (available at www.cmaj.ca/lookup/suppl/doi:10.1503/ cmaj.151365/-/DC1). 


\section{References}

1. Krahn AD, Healey JS, Chauhan V, et al. Systematic assessment of patients with unexplained cardiac arrest: Cardiac Arrest Survivors With Preserved Ejection Fraction Registry (CASPER). Circulation 2009;120:278-85.

2. Gollob MH, Blier L, Brugada R, et al. Recommendations for the use of genetic testing in the clinical evaluation of inherited cardiac arrhythmias associated with sudden cardiac death: Canadian Cardiovascular Society/Canadian Heart Rhythm Society joint position paper. Can J Cardiol 2011;27:232-45.

3. Krahn AD, Sanatani S, Gardner MJ, et al. Inherited heart rhythm disease: negotiating the minefield for the practicing cardiologist. Can J Cardiol 2013;29:122-5.

4. Zipes DP, Link MS, Ackerman MJ, et al. Eligibility and disqualification recommendations for competitive athletes with cardiovascular abnormalities: Task Force 9: arrhythmias and conduction defects: a scientific statement from the American Heart Association and American College of Cardiology. J Am Coll Cardiol 2015;66:2412-23.

5. Sy RW, van der Werf C, Chattha IS, et al. Derivation and validation of a simple exercise-based algorithm for prediction of genetic testing in relatives of LQTS probands. Circulation 2011;124:2187-94.

Affiliations: Division of Cardiology (Dorian), St. Michael's Hospital, and Department of Medicine (Dorian), University of Toronto, Toronto, Ont.; Heart Rhythm Services (Krahn), Division of Cardiology, University of British Columbia, Vancouver, BC
Contributors: Both authors contributed substantially to the conception and design of the manuscript, drafted and revised it critically for important intellectual content, approved the final version to be published and agreed to act as guarantors of the work.

Decisions is a series that focuses on practical evidence-based approaches to common presentations in primary care. The articles address key decisions that a clinician may encounter during initial assessment. The information presented can usually be covered in a typical primary care appointment. Articles should be no longer than 650 words, may include one box, figure or table and should begin with a very brief description (75 words or less) of the clinical situation. The decisions addressed should be presented in the form of questions. A box providing helpful resources for the patient or physician is encouraged. 